Folia Histórica del Nordeste, $\mathbf{N}^{\circ} 19$ (Resistencia, 2011) IIGHI, IH - CONICET, UNNE

\title{
ANÁLISIS CUANTITATIVO DE LA SEGREGACIÓN POR POBREZA EN EL NÚCLEO GRAN RESISTENCIA-GRAN CORRIENTES. UNA MIRADA A TRAVÉS DEL ÍNDICE DE PRIVACIÓN MATERIAL DE LOS HOGARES, 2001.
}

\author{
Quantitative Analysis of Poverty Segregation in Gran Resistencia and Gran \\ Corrientes. A Look through the Privation Index of Households. 2001
}

\section{Aníbal M. Mignone y María Alejandra Fantin}

\section{Resumen}

El Índice de Privación Material de los Hogares (IPMH) utilizado en el Censo Nacional de Población y Vivienda de 2001, es un indicador complejo que permite medir las privaciones de los hogares con diferentes grados de desagregación geográfica. Asimismo, al aplicarlo en las ciudades del Gran Resistencia y Gran Corrientes, muestra las diferencias existentes según los tipos de privaciones y las disparidades en la distribución espacial del fenómeno en ambos ámbitos urbanos.

El trabajo propone una comparación cuantitativa de la segregación por pobreza en el Gran Resistencia y el Gran Corrientes utilizando como principal fuente el IPMH. Se utilizan indicadores que miden diferentes dimensiones de la segregación, para reconocer si existe una desigual distribución de los hogares pobres, si los hogares carenciados se distribuyen en unidades espaciales inmediatamente contiguas y por ende qué ciudad tendría una mayor concentración de áreas segregadas por pobreza.

Con esta investigación, se planea contribuir al conocimiento de la segregación por condiciones de pobreza, en ciudades intermedias del Nordeste Argentino.

$<$ Segregación por pobreza $><$ Índice de Privación Material de los Hogares $><$ Ciudades intermedias $>$

\begin{abstract}
The Index of Household Material Privation (IPMH) used in the National Census of Population and Housing 2001 is a complex indicator to measure deprivation of households in different geographical disaggregation degrees. Likewise, when applied in the cities of Gran Resistencia and Gran Corrientes, it shows the differences by type of deprivation and disparities in the spatial distribution of the phenomenon in both urban areas.
\end{abstract}

The paper proposes a quantitative comparison of poverty segregation in the Gran Resistencia and the Gran Corrientes using as main source the IPMH. Several indexes are used to measure different 
dimensions of segregation, to recognize if there is an unequal distribution of poor households, and if they are distributed in spatial contiguous units, and thus which one of the cities would have a greater concentration of segregated areas for poverty.

With this research, we plan to contribute to the further knowledge of poverty segregation in intermediate cities in Northeast Argentina.

$<$ Segregation by poverty $><$ Households Material Privation Index $><$ Intermediate cities $>$

\section{Introducción}

El estudio de la segregación ha suscitado el interés de diferentes disciplinas como ser la geografía urbana y la sociología; sin embargo, la conceptualización es muy diferente según el campo disciplinar. "En términos sociológicos, segregación significa la ausencia de interacción entre grupos sociales. En un sentido geográfico, significa desigualdad en la distribución de los grupos sociales en el espacio físico. La presencia de un tipo de segregación no asegura la existencia de otro" (Rodríguez Vignoli, 2001:11).

Según Rodríguez y Arraigada (2004:6), la desigual distribución de grupos sociales en el espacio urbano da cuenta de la presencia de la segregación residencial que se manifiesta de diversas maneras: a) por la proximidad física entre los espacios residenciales de los diferentes grupos sociales; b) la homogeneidad social de las distintas subdivisiones territoriales en que se puede estructurar una ciudad, y c) la concentración de grupos sociales en zonas específicas de la ciudad.

Asimismo, el enfoque presenta diferencias de acuerdo con los lugares dónde se lo analice; así, por ejemplo, la segregación de tipo residencial se asocia en el mundo anglosajón con espacios culturales vinculados a cuestiones étnicas y migratorias; por lo tanto el parámetro de medición pasa especialmente por razones asociados a fenómenos raciales. En cambio, en las ciudades latinoamericanas, la segregación residencial hace referencia a los vínculos territoriales entre los estratos socioeconómicos, considerando la concentración de grupos que tienen características socioeconómicas similares, por lo tanto, está más relacionado con los estudios de concentración espacial de la pobreza.

En su conceptualización, se puede considerar que la segregación es el grado de proximidad espacial o la aglomeración geográfica de familias u hogares de una misma categoría social (cf. Sabatini, 2000). Por lo tanto, remite a las diferencias o desigualdades sociales que existen en el espacio físico, donde los sujetos se encuentran separados en categorías que presentan cierto grado de distinción jerárquica o valorativa. A ello, se suma el hecho de existir una ausencia o escasez relativa de mezclas socioeconómicas dentro de las unidades territoriales de la ciudad (cf. Rodríguez Vignoli, 2001). De esta forma, el concepto de segregación apunta a un fenómeno espacial que es independiente de la distribución del ingreso y de las diferencias sociales, por mucho que la segregación residencial esté relacionada con una y otras o que pueda incidir en ellas, por ejemplo contribuyendo a aumentar las desigualdades (Sabatini y Sierralta, 2006). 
El presente trabajo tiene como objetivo realizar una indagación empírica mediante el análisis de la segregación residencial de los hogares pobres localizados en los aglomerados del Gran Resistencia y Gran Corrientes para el año 2001, a fin de reconocer las diferencias que existen entre ambos aglomerados y al interior de los mismos.

En el núcleo bipolar del Gran Resistencia-Gran Corrientes ${ }^{1}$ la pobreza ha ido aumentando progresivamente y si bien no alcanza los valores absolutos de aglomerados como el Gran Buenos Aires, el Gran Córdoba o el Gran Rosario, su manifestación es importante en ambas ciudades, que muestran una pobreza notoria con grandes conjuntos de población que tienen privaciones, un déficit estructural en las viviendas y ausencia de los servicios básicos.

\section{Los índices de segregación y sus dificultades metodológicas}

De acuerdo con Massey y Denton (1988), la segregación se manifiesta en dimensiones como: igualdad, concentración y agrupamiento. Para cada dimensión existe al menos un indicador básico de medida y a través de su aplicación empírica se demuestra estadísticamente que las medidas obtenidas por los diferentes indicadores son independientes entre sí (Lavía Martínez, 2008).

Para el análisis, se han utilizado los siguientes índices:

\begin{tabular}{|l|l|}
\hline * Índices de igualdad & $\begin{array}{l}\text { - Índice de Segregación (IS) } \\
\text { - Índice de Disimilitud de Duncan (ID) } \\
- \text { Îndice de Desigualdad Corregido por la longitud de frontera } \\
\left(\mathrm{D}_{\mathrm{w}}\right) \\
\text { - Índice de Desigualdad Corregido por la forma (D) }\end{array}$ \\
\hline * Índices de concentración & - Índice Delta (DEL) \\
\hline *Índicesdeautocorrelación & $\begin{array}{l}\text { - Índice de Morán Global (I) } \\
\left.\text { - Índice de Morán Local ( } \mathrm{I}_{\mathrm{i}}\right)\end{array}$ \\
\hline
\end{tabular}

Estos índices, más factibles de calcular en ámbitos urbanos, cuentan con una serie de inconvenientes metodológicos que se deben tener en cuenta al efectuar las mediciones. Una de las cuestiones va asociada con el tipo de unidad territorial sobre la que se realiza la medición, porque aunque se comparen las ciudades utilizando la misma unidad censal, en cada aglomerado varía el número de unidades, el tamaño de las mismas y por lo tanto, el grado de homogeneidad interna de la variable utilizada. En consecuencia, se acepta que la elección de un tipo de unidad censal elegida para medir la segregación en un caso concreto, afecta los resultados y la variabilidad de las

\footnotetext{
${ }^{1}$ Las ciudades del Gran Resistencia y del Gran Corrientes forman el núcleo metropolitano bipolar más importante del sistema urbano regional del nordeste argentino (Roccatagliata y Beguiristain, 1992:331).
} 
medidas que dan los índices. El efecto principal sería que cuanto más pequeñas y/o más internamente homogéneas son las unidades, mayores niveles de segregación muestran los índices (cf. Lavía Martínez, 2008). Asimismo, en el caso de analizar solamente una ciudad, es conveniente restringir la información a sólo una escala espacial (fracción, radio censal, barrio, etc.) ya que los resultados varían considerablemente con el uso de diferentes unidades territoriales (cf. Sabatini y Sierralta, 2006).

Asimismo, cada dimensión de la segregación puede presentarse en distinto grado y la combinación de las mismas varía en función del tipo de segregación que se trate y del ámbito y nivel en que se mida. Es decir, las diversas formas en que puede manifestarse la segregación de un grupo que está "aislado" son muchas y complejas. Por último, la interpretación de los resultados requiere no solo el conocimiento de lo que se mide, también hay que considerar el ámbito y la realidad concreta que se pretende medir, ya que formalmente no existe una escala de interpretación preestablecida universalmente que permita definir a partir de qué valores un índice de segregación reconoce condiciones de desigualdad territorial (cf. Lavía Martínez, 2008).

A pesar de las limitaciones mencionadas, se considera que los resultados obtenidos en esta investigación exploratoria, permiten dar una aproximación cuantitativa de las condiciones de segregación de los hogares pobres localizados en dos capitales del nordeste argentino.

La información estadística, inicialmente fue normalizada con el uso del software REDATAM+SP desarrollado por CELADE- CEPAL que permite procesar la información contenida en bases de datos comprimidas y muy extensas, como las provenientes de censos de población completos ${ }^{2}$ y se procesaron los datos a nivel de radio censal, máximo grado de desagregación espacial con que cuenta la fuente de información. Posteriormente, se efectuaron los cálculos de los índices de igualdad y concentración utilizando el software Segregation, mientras que la autocorrelación global y local se obtuvo por medio del Geoda $0.9 .5^{3}$.

\section{Caracteres de la pobreza en el núcleo bipolar y su medición a través del IPMH}

El fenómeno de la pobreza ha tenido en la región del Nordeste argentino (NEA), fuertes manifestaciones tanto en las áreas rurales como en los ámbitos urbanos, sin embargo la pobreza urbana aparece actualmente como uno de las consecuencias más evidente de los efectos negativos del modelo económico vigente en el mundo. La pobreza urbana parece reflejar las propias características de la economía actual y sus modos de producción (Morley, 1998), al tiempo que manifiesta sus rasgos sociales debido a que impide la integración de grupos de activos en el propio sistema productivo, y también deja su impronta en el espacio urbano de las ciudades.

\footnotetext{
${ }^{2} \mathrm{~A}$ través de los microdatos (en este caso datos de los hogares pobres), se pueden generar diversas tabulaciones para cualquier área geográfica definida por el usuario.

${ }^{3}$ Ambos programas permiten trabajar la información contenida a través de los Sistemas de Información Geográfica (SIG).
} 
A pesar de las diferencias en su contexto geográfico y de los disímiles procesos históricos del conjunto Gran Resistencia-Gran Corrientes, se pueden identificar ciertas semejanzas en cuanto a la evolución de la pobreza, similitudes que son más evidentes a partir de la segunda mitad de siglo XX, cuando ambas capitales provinciales experimentaron un fuerte crecimiento, fundamentalmente por el aporte de población procedente de la migración rural y de núcleos urbanos menores ante los sucesivos períodos de crisis en las actividades agropecuarias y el resentimiento en las estructuras económicas provinciales.

El fenómeno de la migración rural-urbana fue una de las principales formas de crecimiento de la población en estas capitales, y aparece relacionado con el aumento de los barrios marginales o asentamientos informales, a través de la modalidad de ocupación individual y colectiva de los terrenos, sumados a procesos de movilidad territorial o de relocalización espacial intraurbana, que también contribuyen a la expansión territorial (Mignone, 2008). Esto da lugar a las ocupaciones de terrenos en la periferia o áreas de expansión de las ciudades capitales, transformándose en verdaderos "bolsones de pobreza".

Por otra parte, la instalación en los espacios que se encontraban suburbanizados o directamente sin urbanizar, va a generar la especulación de las compañías inmobiliarias, que provocan la ocupación a efectos de promover la posibilidad de expropiación de los terrenos (Norte, 26/11/94:13). Indudablemente, con la toma de tierras el valor del terreno ocupado se incrementa, significando notorias ganancias para las compañías. Particularmente, en el último decenio ha aumentado el número de ocupaciones informales en terrenos privados, generando verdaderos conflictos entre los propietarios legales, los nuevos ocupantes y los organismos estatales.

Por lo tanto, en las dos capitales se intensifican los contrastes entre los distintos estratos económicos y entre los grupos localizados en áreas periféricas con respecto a las zonas centrales. Esta segmentación espacial va asociada con el incremento de la segregación residencial, fenómeno que actúa como mecanismo de reproducción de la desigualdad social y económica porque "aísla a los pobres" debido a que estos grupos al convivir con la pobreza y tener pares pobres limitan sus horizontes de posibilidades (cf. Sabatini, 2000). No obstante, también puede existir una segregación no forzada, dónde la población de un grupo social tiende a agruparse con el objeto de preservar su identidad y sus caracteres culturales ${ }^{4}$.

Para poder reconocer las condiciones de pobreza, se utiliza el Índice de Privación Material de los Hogares (IPMH), indicador que permite medir las privaciones

\footnotetext{
${ }^{4}$ Por ejemplo, la segregación residencial de tipo socioeconómica tiene una faceta positiva, vinculado con dos perspectivas: identitaria y funcional. Con la primera representación, se hace referencia al hecho que las personas conviven con vecinos o "pares" a fin de preservar un conjunto de rasgos que forman parte del "ser profundo" de las personas. Desde la situación funcional, se plantea que al residir con personas de la misma condición social, se accede a "beneficios comunes", pues se reducen los costos de transacción al basarse la interacción en códigos de comportamiento y comunicación compartidos (Sabatini 2000, Sabatini et. al. 2001; Rodríguez Vignoli 2001).
} 
de la población partir de datos censales, identificando diferentes grados de carencia en los hogares.

La variable IPMH, utilizada a partir del censo 2001, reconoce a los hogares ${ }^{5}$ según su situación respecto a la privación material considerando dos dimensiones: Patrimonial y Recursos Corrientes. La incapacidad de los hogares para proveerse de uno u otro tipo de recursos es lo que distingue entre los hogares con privación o sin ella. El primer aspecto se vincula a la privación patrimonial que afecta a los hogares en forma más estable y dada su característica de persistencia se lo considera de tipo estructural o crónico. En cambio, la privación de recursos corrientes puede variar considerablemente en el corto plazo y está ligada más directamente a las fluctuaciones de la economía ${ }^{6}$.

La combinación de estas dimensiones define cuatro grupos de hogares: aquellos que no tienen ningún tipo de privación y tres grupos diferenciados según el tipo de privación que presentan: sólo de recursos corrientes, sólo patrimonial y convergente ${ }^{7}$.

- Sin Privaciones (SP)

- Privación solo de Recursos Corrientes (PR)

- Privación solo de Recursos Patrimoniales (PP)

- Privación Convergente (PC)

El análisis relativo del IPMH para la capital chaqueña y correntina, indica un mayor grado de pobreza por ingresos para el Gran Corrientes, mientras que las privaciones de tipo estructural y la pobreza extrema ${ }^{8}$ son superiores en el Gran Resistencia. (cuadro $\mathrm{N}^{\mathrm{o}}$ 1). Fundamentalmente, la localización está asociada con los hogares situados en la periferia de ambos aglomerados, vinculados a los principales núcleos de expansión urbana, especialmente en barrios de reciente instalación, con viviendas deficitarias, sin acceso a los servicios y con población que en su gran mayoría corresponde a trabajadores cuentapropistas o con empleos informales. Gran parte de estos conjuntos reciben asistencia a través de los planes sociales, pero los ingresos son inferiores a la línea de pobreza y a la de indigencia (Mignone, 2008).

\section{Cuadro 1}

\section{IPMH en valores relativos según tipo de privación. Capitales del NEA, 2001}

\begin{tabular}{|l|c|c|c|c|c|}
\hline & SP & PR & PP & PC & Total de hogares \\
\hline Gran Ctes. & 50,67 & 24,11 & 9,72 & 15,50 & 38.400 \\
\hline Gran Rcia. & 46,67 & 18,50 & 11,89 & 22,94 & 48.127 \\
\hline
\end{tabular}

Fuente: Elaboración propia según INDEC. Censo 2001

\footnotetext{
${ }^{5}$ Se entiende por hogares a los grupos de personas que viven bajo el mismo techo y comparten los gastos de alimentación. Se excluyen las instituciones colectivas donde las personas viven bajo un régimen no familiar.

${ }^{6}$ Para un análisis más detallado sobre la construcción del índice, ver los trabajos de Álvarez et. al. (2001); Álvarez (2002); Gómez et. al. (2003).

${ }^{7}$ La identificación de las personas en cierta categoría se establece a partir de las características del hogar al que pertenecen.

${ }^{8}$ A la pobreza convergente se la denomina pobreza extrema por tener las dos dimensiones del IPMH.
} 
El Gran Corrientes tendría menores privaciones y el Gran Resistencia sería la ciudad con mayores índices de pobreza. Sin embargo, los resultados no revelan el grado de concentración espacial de hogares carenciados en ambas capitales, fenómeno que se intenta estudiar a través de las diferentes dimensiones de la segregación.

\section{La dimensión de igualdad}

Un primer paso para medir la segregación, es por intermedio de los índices de igualdad. Inicialmente se utilizaron el índice de Segregación (IS) y el índice de Disimilaridad de Duncan (ID). Ambos varían entre cero y uno, valores que corresponden respectivamente a una distribución exactamente igualitaria y una distribución de máxima segregación. El valor de estos índices se puede interpretar como la proporción del grupo minoritario que tendría que cambiar de residencia o ser relocalizado para obtener una distribución igualitaria en las unidades territoriales (Massey y Denton, 1998). La diferencia radica en que el IS compara la diferencia entre la proporción de individuos del grupo minoritario y la proporción del resto de población, mientras que el ID compara proporciones de dos grupos y no del grupo respecto al resto.

Sin embargo, tanto el IS como el ID de Duncan no permiten incorporar el componente espacial en el análisis, entonces los resultados resultan sensibles a la configuración territorial de las subunidades dentro de la ciudad (Martori y Hoberg, 2004). Por ello, surgieron otros indicadores que incluyen la información espacial para poder calcularlo, como el Índice de Desigualdad corregido por la Longitud de Frontera y el Índice de Desigualdad corregido por la Forma, desarrollados a partir del uso de los sistemas de información geográfica (SIG) y con la disponibilidad de contar con datos de población georreferenciados.

El Índice de Desigualdad Corregido por la Longitud de Frontera $\left[\mathrm{D}\left({ }_{\mathrm{w}}\right)\right]$, tiene en cuenta la diferencia de proporciones del grupo minoritario en las unidades de la ciudad que son contiguas, es decir que es sensible a la presencia de clusters de subáreas que tienen una composición social similar. Pero también considera que las posibilidades de interacción entre los grupos residentes en unidades espaciales contiguas dependen de la longitud de la frontera que separa a las unidades vecinas, interacción entre los individuos que se supone proporcional a la longitud de frontera común (Wong 1993, 1999). En este índice subyace la idea de que la interacción entre unidades disminuye el nivel de segregación expresado en términos de la Disimilitud.

Al incorporar los elementos de configuración espacial, también se debe considerar la forma geométrica de los radios censales y por ello se utiliza el índice de Desigualdad corregido por la Forma $\left[\mathrm{D}\left(\mathrm{S}_{\mathrm{s}}\right)\right]$. En este caso, la forma de los radios afecta la probabilidad de interacción entre individuos de diferentes grupos en unidades vecinas (Wong, 1993, 1999). La medida más sencilla para el cálculo de la forma, es la relación entre perímetro y área; por lo tanto el cociente entre estas dos magnitudes determina lo que se denomina compacidad de la unidad. La compacidad es mínima cuando el cociente es máximo y a más compacidad menos probabilidad de interacción (Martori y Heberg, 2004). 
Cuadro 2

\section{Índices de igualdad según tipo de IPMH. Gran Resistencia y Gran Corrientes. Año 2001}

\begin{tabular}{|c|c|c|c|c|c|c|}
\hline \multirow{2}{*}{} & \multicolumn{2}{|c|}{ Rec. Ctes. (PR) } & \multicolumn{2}{c|}{ Patrimonial (PP) } & \multicolumn{2}{c|}{ Convergente (PC) } \\
\cline { 2 - 7 } & G. Rcia. & G. Ctes. & G. Rcia. & G. Ctes. & G. Rcia. & G. Ctes. \\
\hline IS & 0,249 & 0,218 & 0,363 & 0,379 & 0,513 & 0,500 \\
\hline ID & 0,282 & 0,227 & 0,529 & 0,476 & 0,629 & 0,574 \\
\hline $\mathrm{D}_{\mathrm{w}}$ & 0,214 & 0,169 & 0,461 & 0,418 & 0,560 & 0,517 \\
\hline $\mathrm{D}_{\mathrm{s}}$ & 0,256 & 0,198 & 0,503 & 0,446 & 0,602 & 0,545 \\
\hline
\end{tabular}

Fuente: Elaboración propia según INDEC. Censo 2001.

Los índices de igualdad (Cuadro 2) revelan que la segregación aumenta conforme varía el tipo de pobreza, siendo los hogares con Privación Convergente (PC) los que tienen una mayor concentración espacial en ambas capitales, ya que es necesario redistribuir más del $50 \%$ de los hogares con pobreza extrema para que no exista segregación. Por lo tanto, los hogares con carencia extrema tienden a estar más segregados en el espacio urbano, lo que presuponemos, se asociaría a una segregación impuesta por las condiciones socioeconómicas.

En cambio, la Privación por Recursos Corrientes (PR) segregaría menos que la Privación Patrimonial (PP) probablemente por el fenómeno que miden: la primera está vinculada con el ingreso percibido en los hogares, por lo tanto con situaciones coyunturales que son dinámicas y fluctuantes; mientras que la segunda está asociada con la vivienda, componente estructural sujeto a cambios lentos en el tiempo y el territorio.

Otra observación de los datos permite conocer que los resultados de los índices espaciales son menores que los de la variante no espacial, debido a la corrección introducida. Esta situación indica que el nivel de segregación se puede considerar sobrevalorado cuando los cálculos se basan únicamente en los indicadores no espaciales (Martori y Hoberg, 2004). Sin embargo, como los resultados más próximos al ID de Duncan corresponden al índice corregido por la forma $\left(\mathrm{D}_{\mathrm{S}}\right)$, más completo por los elementos espaciales que incorpora, la sobreestimación puede ser menor que la percepción inicial.

Una primera comparación según el índice IS, revela que el Gran Corrientes tiene un menor grado de segregación, especialmente en la privación por recursos corrientes y la convergente (cuadro 2). Esta condición es relevante, porque el cuadro 1 indicaba que el aglomerado tiene el mayor porcentaje de hogares con privación de recursos corrientes, pero la distribución de estos hogares sería menos concentrada en el interior de la ciudad 9 .

\footnotetext{
${ }^{9}$ No obstante, es probable que puedan existir grupos de hogares pobres separados entre los radios censales que de forma agregada, hacen desaparecer la segregación individual del grupo.
} 
Por su parte, la segregación por pobreza convergente es alta para el Gran Resistencia, y posee una de las "peores periferias" del NEA (Mignone, 2008), debido a la fuerte intensidad de la pobreza y la alta concentración de los hogares con privación convergente en los bordes del ejido urbano resistenciano.

Con los índices de Disimilaridad de Duncan, el D corregido por la longitud de Frontera y D corregido por la forma, se han comparado los hogares según el tipo de privación con respecto al conjunto que no tiene privaciones. En el Gran Corrientes, la segregación se reduce en la pobreza por ingreso, más aún con los índices espaciales, lo que estaría dando muestra de una "mayor igualdad" en la distribución espacial entre los hogares pobres y no pobres de la ciudad, por lo menos con los resultados obtenidos a nivel de los radios censales.

El Gran Resistencia mantiene una diferencia superior al 5\% según los tipos de privaciones (aún con los índices espaciales), y está marcando condiciones de fuerte segregación o "hipersegregación" en la pobreza convergente, porque los valores superan el 60\% de concentración (Massey 1985). Se considera nuevamente que esta situación se asocia a una condición de segregación impuesta por la falta de medios socioeconómicos de los hogares, los que se localizan primordialmente en la periferia norte y sur de Resistencia, así como en el extremo norte de Barranqueras, el norte y suroeste de Fontana (Mignone, 2008). Estos espacios contienen un importante número de asentamientos informales, en terrenos anegables periódicamente y con déficit ambiental.

\section{La concentración de los hogares con privaciones}

Los indicadores de concentración permiten conocer acerca de la ocupación de un espacio físico (superficie) por parte de un grupo de población en el medio urbano. En este caso, cuanto menor sea la porción de espacio urbano ocupado por un grupo, más concentrado y segregado se encuentra. Por lo tanto, es una medida que considera la densidad relativa del grupo en las unidades espaciales en relación a su densidad media en el conjunto urbano (Massey 1988).

Para medir la concentración, se utilizó el índice Delta (Duncan, 1961 en Martori y Hoberg, 2004) que calcula la diferencia entre la proporción de la población de un grupo en cada unidad respecto al total del grupo en la ciudad y la proporción de la superficie de cada unidad con el total de la ciudad. También se puede interpretar como el porcentaje de población del grupo X que ha de cambiar de residencia para obtener una distribución uniforme en toda la ciudad.

En el Cuadro 3, el índice Delta de Duncan (Del) marca diferencias importantes entre ambas capitales. El Gran Resistencia supera ampliamente a la capital correntina en la PR y PC, ya que por ejemplo, habría que mover el 57\% de los hogares segregados con pobreza extrema para que no exista segregación debido a que la proporción de este grupo en cada radio censal con respecto al total del grupo en la ciudad es alta. Esto 
está marcando una condición de fuerte concentración espacial de los hogares pobres, que presentan densidades altas en espacios críticos vinculados a los nuevos barrios informales ${ }^{10}$ que rodean la periferia norte y sur de la ciudad, con un bajo nivel educativo de la población y predominantemente con empleos informales o cuentapropistas (Meichtry y Mignone, 2001).

Cuadro 3

Índices de concentración y autocorrelación según tipo de IPMH. Gran Resistencia y Gran Corrientes. Año 2001

\begin{tabular}{|c|c|c|c|c|c|c|}
\hline \multirow{2}{*}{} & \multicolumn{2}{|c|}{$\begin{array}{c}\text { Recursos Corrientes } \\
\text { (PR) }\end{array}$} & \multicolumn{2}{c|}{$\begin{array}{c}\text { Patrimonial } \\
\text { (PP) }\end{array}$} & \multicolumn{2}{c|}{$\begin{array}{c}\text { Convergente } \\
\text { (PC) }\end{array}$} \\
\cline { 2 - 7 } & G. Rcia. & G. Ctes. & G. Rcia. & G. Ctes. & G. Rcia. & G. Ctes. \\
\hline Del & 0,601 & 0,425 & 0,404 & 0,573 & 0,571 & 0,431 \\
\hline Morán & 0,285 & 0,243 & 0,431 & 0,441 & 0,500 & 0,468 \\
\hline
\end{tabular}

Fuente: Elaboración propia según INDEC. Censo 2001.

La mayor segregación de hogares con PP en el Gran Corrientes, indica que los hogares residentes en viviendas deficientes tienen una compacidad menor según los radios censales, revelando una mayor densidad de esta privación en las unidades territoriales.

\section{La autocorrelación espacial y la pobreza en el núcleo Gran Resistencia-Gran Corrientes}

La autocorrelación espacial permite medir la correlación de una variable consigo misma y considera los atributos vinculados con aspectos espaciales. La autocorrelación espacial se basa en el postulado que supone que en el espacio geográfico todo se encuentra relacionado con todo, pero los espacios más cercanos están más relacionados entre sí que con los más alejados de acuerdo con el principio de Tobler (Buzai y Baxendale, 2006).

Para medir la autocorrelación se ha utilizado el Índice de Morán (I), en el cuál los valores van de -1 a 1 . Un índice de 1 indica una autocorrelación espacial positiva, un valor 0 indica un patrón aleatorio y -1 indica una fuerte autocorrelación espacial negativa. El índice de Morán permite vincular en forma conjunta la dependencia entre lugares próximos y valores de variables o atributos que nos interesan (Velázquez y Cepeda, 2004).

Con este índice se puede contrastar la presencia de tendencias o estructuras espaciales generales en la distribución de una variable sobre el ámbito urbano completo,

\footnotetext{
${ }^{10}$ A decir de Sabatini y Sierralta (2006), los grupos pobres son excluidos de ciertas áreas de la ciudad y empujados a aglomerarse en las peores áreas de la ciudad por causa del precio del suelo, las políticas de vivienda social e incluso las erradicaciones forzosas.
} 
logrando conocer la tendencia al agrupamiento o a la dispersión de las unidades espaciales de acuerdo con las variables analizadas ${ }^{11}$.

Las capitales muestran una autocorrelación espacial positiva en las diferentes privaciones, con marcadas diferencias entre las dos ciudades (Cuadro 3). Destacamos el hecho de la alta correlación por privación convergente en el Gran Resistencia (50 \%); por lo tanto, existe una contigüidad espacial en las unidades censales y se formarían "enclaves" con pobreza extrema. Como se dijera anteriormente, la convergencia de situaciones en un mismo espacio puede primordialmente estar sujeta a cuestiones socioeconómicas forzadas (falta de empleo, ingreso bajos), aunque también podría deberse a los mecanismos de preservación de la clase social (Sabatini, 2003) lo que permitiría reconocer que ambas condiciones podrían presentarse en la ciudad ${ }^{12}$.

Asimismo, se puede presuponer que se está produciendo el paso de una segregación de primer tipo (concentración espacial del grupo pero con el espacio compartido con otros grupos) a una de segundo tipo, que se caracterizaría por una homogeneidad de estos espacios, que con el correr del tiempo tienden a aislarse físicamente de otros grupos sociales, mientras se mantienen fuertes otras formas de integración social, como la laboral o la económica (Sabatini, 2003).

En el Gran Corrientes, hay una mayor autocorrelación de la privación patrimonial, asumiendo entonces que la distribución de las viviendas deficientes de Corrientes tiene una mayor contigüidad en las unidades territoriales. De manera opuesta, disminuye el valor de autocorrelación en la pobreza por recursos corrientes lo que demuestra que estos hogares tienen una menor contigüidad espacial y un grado de aleatoriedad en la distribución en las unidades censales. Esta situación, sumada a los menores índices de igualdad y concentración, inicialmente dan pautas de que a nivel de radio censal no habría una fuerte condición de autosegregación de este conjunto de hogares.

Por su parte, el diagrama de dispersión ${ }^{13}$ es una herramienta gráfica fundamental para el análisis de dependencia espacial en una serie y representa una línea de regresión cuya pendiente está formada por las posiciones de cada unidad espacial en el sistema de coordenadas (Buzai y Baxendale, 2006). En el diagrama de dispersión de Moran (cuadro 4), se estandariza la variable a analizar y se obtiene el retardo espacial de dicha variable estandarizada, representándose ambos valores en un eje cartesiano. La pendiente de la recta de regresión es el valor del estadístico I de Moran de autocorrelación espacial

\footnotetext{
$\overline{11}$ La matriz de pesos especiales construida en el Geoda 0.9.5, fue la "matriz de contactos binarios", atendiendo a un criterio de contigüidad de tipo Queen o Reina, con el cuál se concibe que serán vecinas de i las unidades que comparten algún lado o vértice con i.

${ }^{12}$ Los trabajos de Meichtry y Mignone (2001) y Mignone (2003, 2004), con relevamientos realizados en asentamientos informales de la ciudad de Resistencia, indican que los migrantes del interior de la provincia a llegar a la ciudad, se dirigen directamente al asentamiento como consecuencia de las redes sociales (generalmente por parentesco) formadas entre la población migrante y la residente en el barrio informal. No obstante, se entiende que hace falta una mayor comprobación empírica de este fenómeno.

${ }^{13}$ En el eje x aparecen los valores estandarizados de la variable para cada unidad espacial y en el eje y se encuentran los valores estandarizados del promedio de los valores de las unidades espaciales vecinas para la misma variable en el caso de la autocorrelación espacial univariada (Buzai y Baxendale, 2006).
} 
global, de forma que cuanto mayor sea el ángulo que forme ésta con el eje de abscisas, más fuerte será el grado de autocorrelación espacial (Chasco Irigoyen, 2003).

La pendiente de la recta es más inclinada en la privación convergente lo que demuestra una mayor autocorrelación espacial. Asimismo, el IPMH por ingresos corrientes tendría una segregación baja con autocorrelación que no es significativa, diferente a la privación por recursos patrimoniales que refleja una segregación media dónde la autocorrelación es significativa, mientras que en la privación convergente la segregación es alta y la autocorrelación significativa.

Con el I de Morán se obtiene un indicador global de autocorrelación de las variables analizadas, aunque el grado de relación significativo entre éstas y las unidades censales ocurre sólo en determinadas zonas de la ciudad. Para esta cuestión, se recurre al $I_{i}$ de Moran (Anselin, 1995), indicador local de autocorrelación espacial (LISA) que tienen como objetivo que el estadístico obtenido indique la presencia de subáreas de dependencia espacial dentro del espacio general dado ${ }^{14}$.

Con la utilización del software Geoda $0.9 .5^{15}$ se obtienen los clusters con presencia alta de miembros de un grupo, pudiendo identificar zonas con una alta manifestación de hogares pobres rodeados de áreas con presencia también alta de hogares en la misma situación (condición High-High). A decir de Martori y Hoberg (2004), la detección de este tipo de situaciones en el espacio urbano, puede ser de gran utilidad en la aplicación de políticas sociales que eviten los problemas derivados de la segregación.

La descripción de la cartografía del Gran Resistencia ${ }^{16}$ reconoce espacios puntuales con privación por ingresos (plano 1), que coinciden con el sector sur del municipio de Barranqueras, especialmente en los barrios 110, 120, 150 y 200 viviendas y en el ejido municipal de Fontana, con los barrios Cacique Pelayo, Güemes, Belgrano y San Pantaleón. En cambio, en Resistencia se acota a espacios puntuales como la porción meridional de los barrios Mujeres Argentinas y Villa Río Negro.

En el Gran Corrientes, la autocorrelación se acentúa hacia el sur, en los radios localizados entre la avenida Maipú y la ruta nacional $\mathrm{N}^{0} 12$ (barrios J. F. Quintana, Pirayuí y Ciudades Correntinas). Por el norte, en las proximidades del casco central, aparece otro espacio con hogares pobres por ingresos, que coincide con una gran proporción de barrios con estudiantes universitarios provenientes de la región, que no son perceptores de ingresos al ser personas dependientes de recursos ${ }^{17}$.

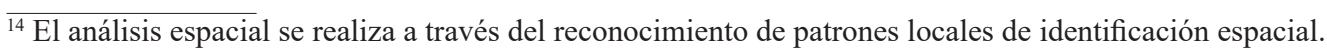
Este método descompone el índice I de Moran y verifica en cuánto contribuye cada unidad espacial a la formación del valor general, permitiendo obtener un valor de significatividad para cada cluster formado por los valores similares de cada unidad espacial y sus vecinos.

${ }^{15}$ Con la opción Map Cluster de Geoda 0.9.5 se observan los lugares estadísticamente significativos según la correlación espacial.

${ }^{16}$ Los planos confeccionados con el Geoda 0.9.5 no tienen escala, porque el software no trae incorporada esta herramienta en el programa.

${ }^{17}$ Los estudiantes perciben estipendios de los padres y entonces el IPMH no los capta de manera adecuada, porque los considera como perceptores de hogar desocupados y sin una remuneración fija (cf. Formiga y Prieto, 2006). 
Folia Histórica del Nordeste, $\mathbf{N}^{\circ} 19$ (Resistencia, 2011) IIGHI, IH - CONICET, UNNE

Cuadro 4. Diagrama de dispersión para la autocorrelación espacial según tipo de IPMH. Gran Resistencia y Gran Corrientes. Año 2001

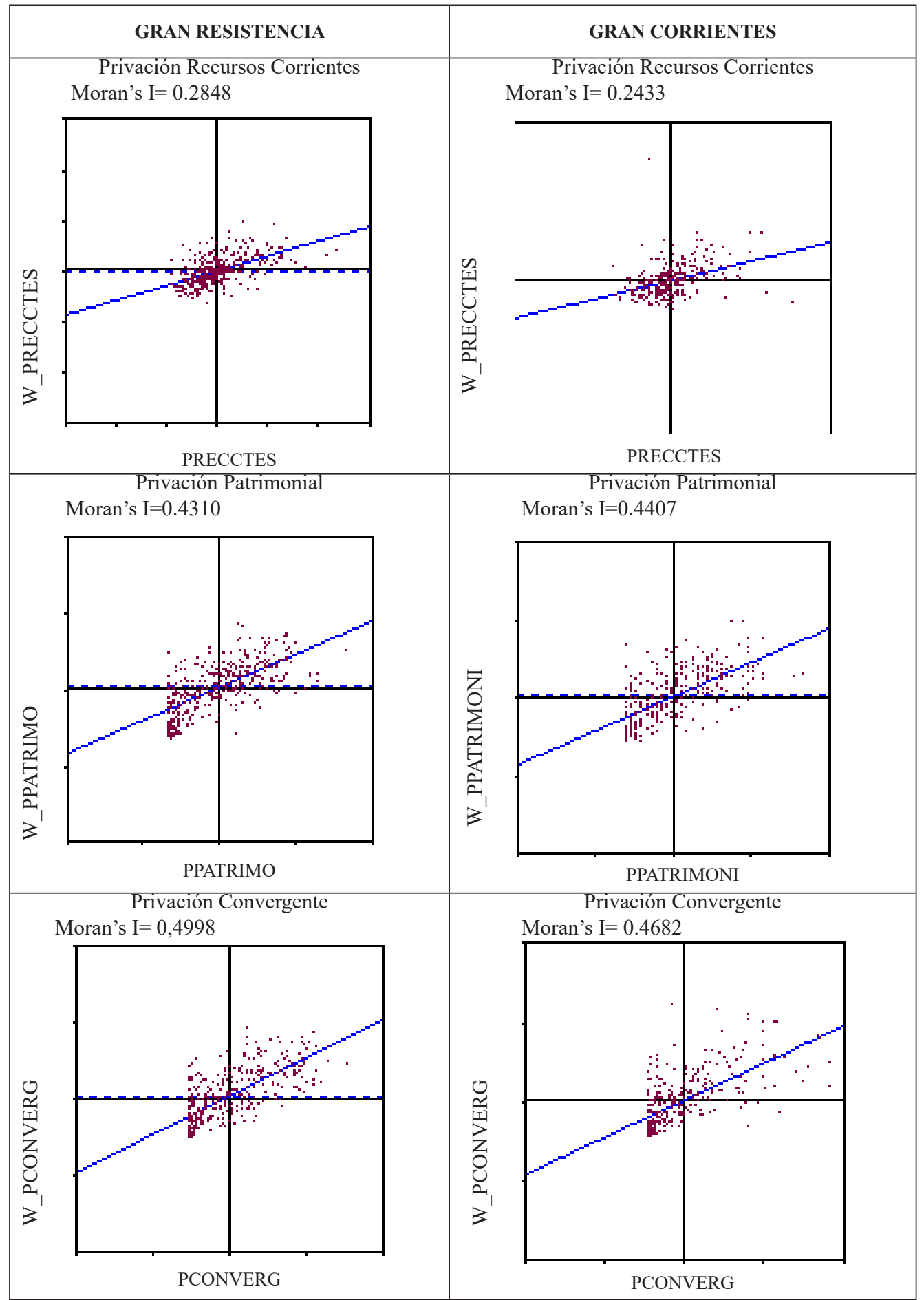

Fuente: elaboración según base de datos REDATAM+SP 2001 
Plano 1

\section{Autocorrelación local: IPMH Recursos Corrientes}

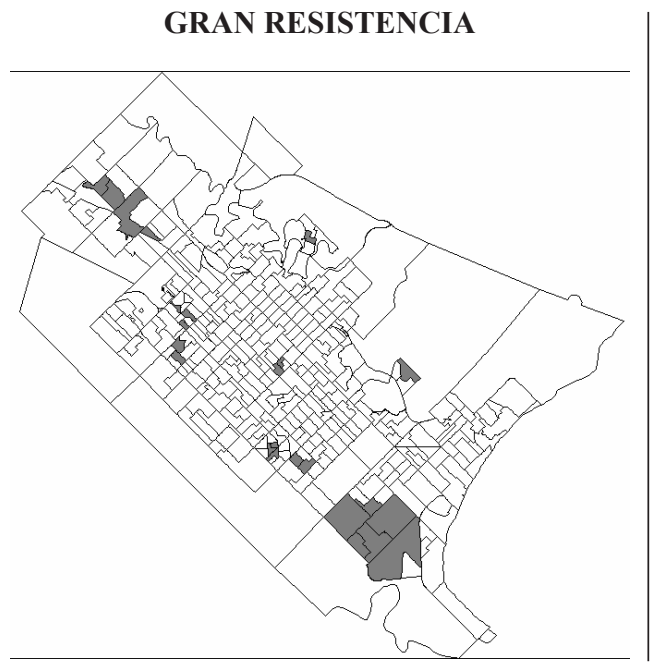

GRAN CORRIENTES

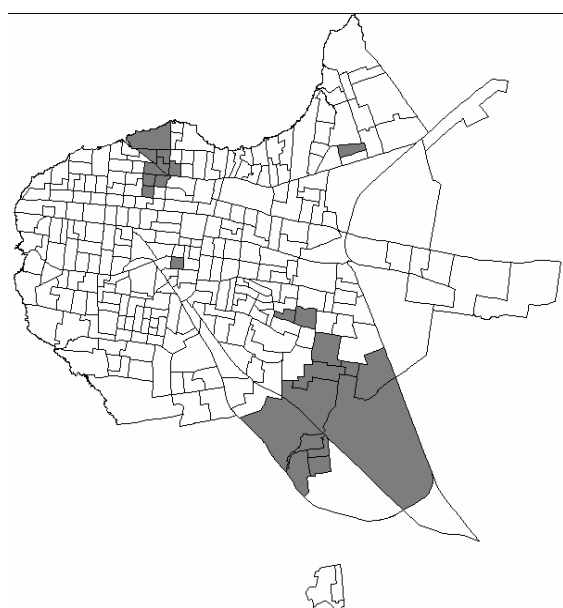

Fuente: Elaboración según INDEC. Censo 2001.

El Moran local correspondiente a la privación solo de recursos patrimoniales (plano 2), reconoce tres espacios en el Gran Resistencia. Se observan: toda la periferia norte del aglomerado, que coincide especialmente con los barrios Don Santiago, Parque Autódromo, Villa Inmaculada, Villa Oro; otros lugares caracterizados por viviendas construidas con materiales precarios, provisión de agua por canillas públicas o fuera de las viviendas y deficiencias sanitarias. Y, finalmente, caracteres similares se encuentran en los barrios de todo el cuadrante sur-suroeste (Vista Linda, Menem Junior, Villa Facundo, Don Andrés, Villa Libertad, entre otros) y del noreste (asentamientos Vuelta de Obligado y Padre Fyrnys entre los más representativos) de Resistencia.

En Corrientes, la privación patrimonial coincide con los núcleos históricos de pobreza localizados en el extremo norte de la aglomeración (barrios Lomas del Mirador, Apipé); en los radios localizados al este de la ruta nacional $\mathrm{N}^{\circ} 12$, concurrente con los núcleos de expansión de la ciudad que se prolongan por la ruta provincial $\mathrm{N}^{\circ} 5$; por el cuadrante sur - suroeste aparecen en los barrios Ongay, Nuestra Sra. de Guadalupe, San Antonio, Concepción, San Roque, así como las proximidades del área denominada "La Arenera" (Patono) y cerca de "Astilleros Corrientes" (barrios Juan Vera y Galván).

En cuanto a la pobreza convergente (Plano 3), la autocorrelación se acentúa más en toda la periferia del Gran Resistencia, conformando un cinturón que principalmente envuelve la ciudad por todo el cuadrante sur-sureste. A la distribución del mapa anterior, se agrega todo el sector norte de Barranqueras (Villa María Cristina, La Toma y Villa Eva María), la parte norte de Fontana, así como el municipio de Puerto Vilelas ${ }^{18}$.

\footnotetext{
${ }^{18}$ Aquí realizamos una acotación: los radios de los extremos sur y del norte del Gran Resistencia tienen una gran superficie; entonces la representación cartográfica de las variables pueden generar un fuerte impacto visual de la segregación y ofrecer una lectura distorsionada al lector.
} 
Plano 2

\section{Autocorrelación local: IPMH Recursos Patrimoniales}
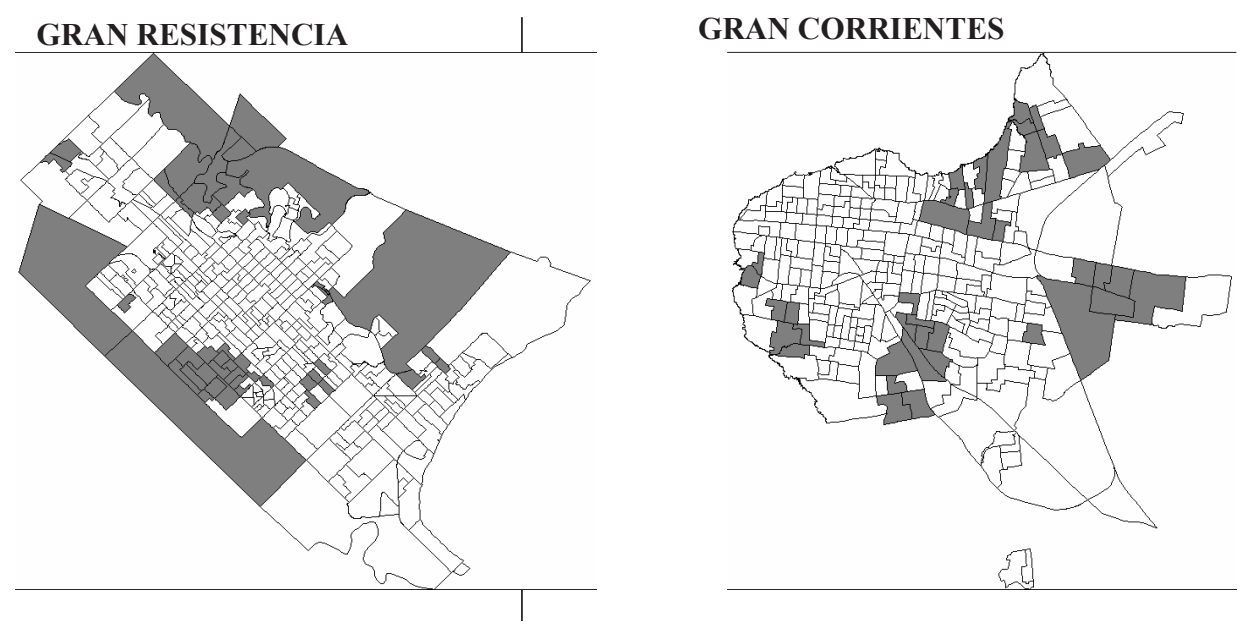

Fuente: elaboración según INDEC. Censo 2001.

En el Gran Corrientes, los radios con alta autocorrelación se localizan por el norte, coincidiendo con los barrios Plácido Martínez, Pujol, Quinta Ferré; por el este de la ruta nacional No 12 en los barrios Cremonte, San Ignacio, José Ponce; y por el sur lo encontramos en los barrios Irupé, Paloma de la Paz, Ongay, San Roque y Patono.

Plano 3

\section{Autocorrelación local: IPMH Convergente}

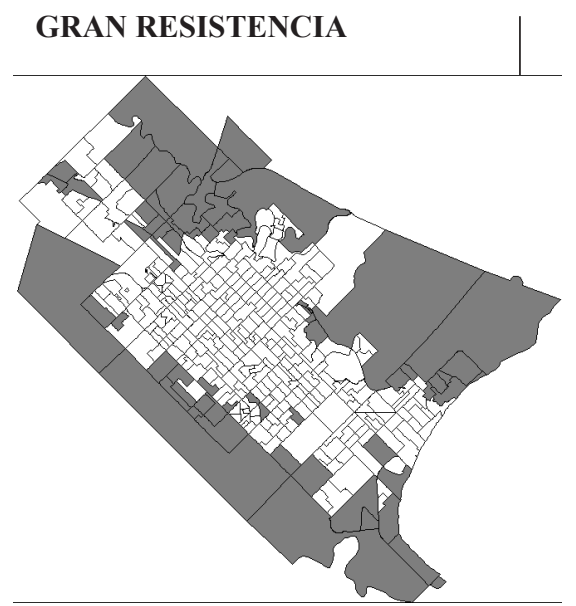

\section{GRAN CORRIENTES}

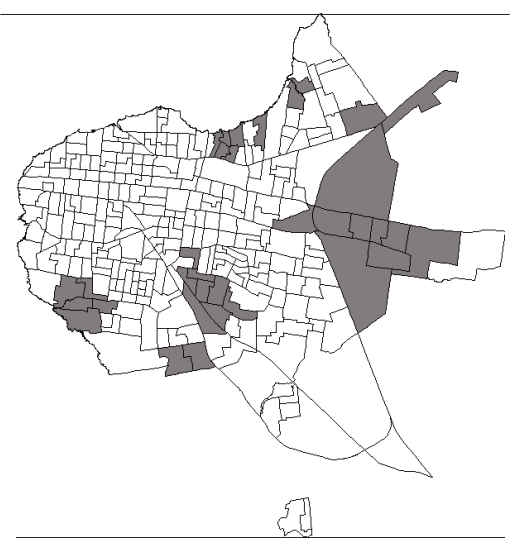

Fuente: elaboración segúnINDEC. Censo 2001. 


\section{Conclusiones}

Según Rodríguez Vignoli y Arraigada (2004:19) en las ciudades latinoamericanas "la segregación se perfila como un mecanismo particularmente importante en la reproducción de las desigualdades socioeconómicas, el aislamiento de los pobres y la inseguridad ciudadana, especialmente para los pobres".

En los aglomerados analizados se puede concluir que no existe, espacialmente, una distribución equilibrada de los hogares con y sin privaciones; por el contrario, las diferencias son marcadas en los dos ejidos, condición que se acentúa aún más a medida que el grado de privación se acrecienta.

Por lo tanto, una primera apreciación permite reconocer que en ambas capitales, según los cálculos realizados, hay un incremento de la segregación por pobreza según aumenta el grado de privación. Esto revela que habría una condición de alta segregación relacionada con las condiciones socioeconómicas de la población, dónde los hogares con extrema pobreza estarían agrupados y situados en los bordes de los ejidos urbanos, sin servicios básicos y con amplio déficit ambiental.

De igual manera, la autocorrelación espacial crece positivamente a medida que la privación se intensifica, dando lugar a enclaves o zonas de contigüidad espacial con pobreza extrema.

Por otra parte, los índices utilizados revelan una importante disparidad entre ambas capitales, no sólo en los niveles de segregación sino también en las modalidades de segregación.

En el Gran Corrientes, los cálculos efectuados con los diferentes índices de igualdad, evidencian una mayor equidad en la distribución espacial de los hogares con y sin privaciones. Por otra parte, la concentración de estos hogares carenciados, no da muestras de un fuerte agrupamiento, por lo menos en lo que respecta a la superficie ocupada en términos de extensión relativa de espacio físico. La autocorrelación espacial es poco significativa, aunque el Moran local estaría mostrando coincidencia con los espacios que históricamente han tenido altos índices de pobreza o con las áreas de expansión del ejido urbano correntino.

En cambio, en el Gran Resistencia la pobreza se manifiesta con mayor intensidad, ya que existe una mayor concentración espacial de los hogares con privaciones. Asimismo se puede observar una mayor densidad de hogares con privaciones por unidad censal y una mayor contigüidad de las privaciones en los radios censales, especialmente en el cinturón o anillo continuo, que rodea al aglomerado por el norte y el sur y que notoriamente coincide con los asentamientos informales, cuya población posee bajos ingresos y amplias dificultades en el acceso al empleo.

Si bien se reconoce que el estudio de la segregación por pobreza requiere de otros tipos de enfoques complementarios que avalen los resultados obtenidos, los índices utilizados permiten reconocer diferentes aspectos y características de la segregación de los hogares carenciados. 
En definitiva, esta investigación supone una primera aproximación al estudio de la segregación por pobreza en las capitales del NEA, condición que seguramente requiere futuros análisis a través de otros parámetros de medición y atendiendo a las particularidades del interior de cada ciudad, situación que no siempre es posible analizar por el grado de desagregación espacial que tiene la información censal.

\section{Referencias Bibliográficas}

Álvarez, Gustavo. 2002. "Capacidad económica de los hogares. Una aproximación a la insuficiencia de ingresos". En: Notas de población $N^{\circ} 74$. CEPAL, Santiago de Chile. pp. 213-250.

Álvarez, Gustavo; Ariel Lucarini y Silvia Mario. 2001. "La pobreza a partir de datos censales: nuevos desarrollos basados en la capacidad económica de los hogares. Censo experimental Pergamino, 1999". VI Jornadas de la Asociación de Estudios de Población de la Argentina. Asociación de Estudios de Población de la Argentina, Neuquén. 14 al 16 de noviembre de 2001. pp.167-184.

Anselin, Luc. 1995. "Local Indicators of Spatial Association-LISA". En: Geographical Analysis. Volumen 27, $\mathrm{N}^{\circ}$ 2. pp. 93-115.

Buzai, Gustavo y Claudia Baxendale. 2006. Análisis socioespacial con Sistemas de Información Geográfica. Lugar Editorial: GEPAMA, Buenos Aires.

CEPAL/PNUD. 1991. Magnitud de la pobreza en América Latina. Naciones Unidas, Santiago de Chile.

Chasco Irigoyen, Coro. 2003. "Métodos gráficos del análisis exploratorio de datos espaciales". Anales de Economía aplicada 2003. No XVII. Asociación Internacional de Economía Aplicada, Almería, España. URL: http://www.asepelt.org/ficheros/File/Anales/2003\%20 \%20Almeria/asepeltPDF/93.PDF

Diario Norte. 2500 familias ocupan ilegalmente los terrenos en Resistencia. 26 de noviembre de 1994. pp. 13-14.

Duncan, Otis. y Duncan, Beverly. 1955. "A methodological analysis of segregation indexes". En: American Sociological Review. Volumen 20. № 2. pp. 210-217.

Formiga, Nidia y María B. Prieto. 2006. "La medición de la pobreza a partir de datos censales. El caso de Bahía Blanca". En: Octavo Encuentro Internacional Humboldt. Colón, Entre Ríos, Argentina. 25 a 29de septiembre de 2006. Versión en CD-Rom.

Gómez, Alicia; Silvia Mario y M. Olmos. 2003. "Índice de Privación Material de los Hogares (IPMH). Desarrollo y aplicación con datos del Censo Nacional de Población y Viviendas 2001". En: VII Jornadas Argentinas de Estudios de Población. AEPA. Universidad Nacional de Tucumán, Tafí del Valle. Tucumán. Tomo II. pp.1001-1017

Katzman, Rubén. 2001. "Seducidos y abandonados. El aislamiento social de los pobres urbanos". En: Revista de la CEPAL N ${ }^{\circ} 76$. pp.171-198.

Lavía Martínez, Cristina. 2008. La segregación residencial de la población extranjera de Bilbao. Revista ACE (Arquitectura, ciudad y entorno). Año III, Número 8, octubre de 2008. URL: http://www.upcommons.upc.edu/revistes/bitstream/2099/6578/.../4_LAVIA.pdf. 
Martori i Cañas, Joan y Karen Hoberg. 2004. "Indicadores cuantitativos de segregación residencial. El caso de la población inmigrante en Barcelona”. En: Geo Crítica / Scripta Nova. Revista electrónica de Geografía y Ciencias Sociales. Vol. VIII, Nº 169. Universidad de Barcelona, Barcelona. 15 de julio de 2004. URL: http://www.ub.es geocrit/sn/sn-169.htm

Martori, Joan C., Karen Hoberg y Rafa Madariaga. 2008. "La incorporación del espacio en los métodos estadísticos: autocorrelación espacial y segregación”. Diez años de cambios en el Mundo, en la Geografía y en las Ciencias Sociales, 1999-2008. Actas del X Coloquio Internacional de Geocrítica. Universidad de Barcelona, Barcelona, España. 26 al 30 de mayo de 2008. URL: http://www.ub.es/geocrit/-xcol/195.htm.

Massey, Douglas y Nancy Denton. 1998. "The dimensions of residential segregation". En: Social Forces. Volumen 67:2. pp. 281-315.

Massey, Douglas. 1985.'Ethnic residential segregation: a theoretical synthesis and empirical review". En: Sociology and Social Research $N^{\circ}$ 69. pp. 315-350.

Meichtry, Norma y Aníbal Mignone. 2001. "Movilidad territorial. Una contribución a partir de la población en asentamientos periféricos espontáneos en Resistencia, Chaco, en la década de 1990". En: VI Jornadas de la Asociación de Estudios de Población de la Argentina. Asociación de Estudios de Población de la Argentina (AEPA) y Universidad Nacional del Comahue, Neuquén. 14 al 16 de noviembre de 2001. pp. 324-339.

Mera, Gabriela y Mariana Marcos. Pensar la espacialidad, medir la espacialidad. Propuestas teóricas y desafíos metodológicos para analizar la distribución y diferenciación en el espacio urbano. Publicaciones de Becarios del Grupo de Estudios Población, Migraciones y Desarrollo. Instituto de Investigaciones Gino Germani, UBA. URL: http://www.iigg. fsoc.uba.ar/pobmigra/archivos/mera_marcos_espacialidad.pd.

Mignone, Aníbal. 2003. La movilidad territorial desde la perspectiva de la población instalada en asentamientos espontáneos en el Gran Resistencia, a principios del siglo XXI. En: VII Jornada Argentina de Estudios de la Población. Asociación de Estudios de Población de la Argentina (AEPA). 5 al 7 de noviembre de 2003. Tafí del Valle, Tucumán, Argentina. pp. 315-328.

Mignone, Aníbal. 2004. Las condiciones de movilidad territorial en la población de los asentamientos espontáneos del 2000, en el Gran Resistencia. En: XXIV Encuentro de Geohistoria Regional $2^{\circ}$ Simposio sobre el estado actual del conocimiento antropológico del Gran Chaco Meridional. Resistencia, 9 a 11 de septiembre de 2004. pp. 391- 401.

Mignone, Aníbal. 2008. "Las condiciones de pobreza en las capitales del nordeste argentino a principios del siglo XXI. Su análisis a través del Índice de Privación Material de los Hogares (IPMH)". En: XXVII Encuentro de Geohistoria Regional. Instituto de Investigaciones Geohistóricas-Conicet. 28-30 de agosto de 2008. Versión en CD-Rom.

Morley, Samuel A. 1998. "El impacto del entorno macroeconómico sobre la pobreza urbana". En: Taller Sobre la Pobreza Urbana en América Latina y el Caribe. 14 al 16 de mayo de 1998, Río de Janeiro. URL: Www.wbln0018.worldbank.org.

Roccatagliata, Juan y Susana Beguiristain. 1992. "Urbanización y sistema urbano”. En: Juan Roccatagliata (coord.). La Argentina. Geografia general y los marcos regionales. 2 da. ed. Editorial Planeta, Buenos Aires. pp. 73-143.

Rodríguez Vignoli Jorge. 2001: “Segregación residencial socioeconómica: ¿qué es?, ¿cómo se 
mide?,¿qué está pasando, ¿importa?”. Serie Población y Desarrollo $N^{o} 16$. Agosto de 2001. CELADE-UNFPA, Santiago de Chile. URL: http://www.eclac.cl/publicaciones xml/8/7888/lcl1576-P.pdf

Rodríguez Vignoli, Jorge y Carlos Arraigada. 2004. "Segregación residencial en la ciudad Latinoamericana”, en: Revista Eure (online). Volumen XXIX, No 89. Santiago de Chile, Chile.

Sabatini Francisco y Carlos Sierralta. 2006. Medición de la Segregación Residencial: Meandros Teóricos y Metodológicos, y Especificidad Latinoamericana. Documento de Trabajo $\mathrm{N}^{\mathrm{o}}$ 38. Instituto de Estudios Urbanos_Facultad de Arquitectura, Diseño y Estudios Urbanos de la Pontificia Universidad Católica de Chile. URL: http://www.ieut.cl/media/archivos/ sabatinisierralta_dt38.pdf.

Sabatini, Francisco, Gonzalo Cáceres y Jorge Cerda. 2001. "Segregación residencial en las principales ciudades chilenas: Tendencia de las tres últimas décadas y posibles cursos de acción". En: Revista EURE (online). Volumen XXVII, No 82:21-42. Santiago de Chile, Chile. URL:http://www.scielo.cl/scielo.php?script=sci_arttext\&pid=S02501612001008200002\&lng=es\&nrm=iso.

Sabatini, Francisco. 2000. "Reforma de los mercados de suelo en Santiago, Chile: efectos sobre los precios de la tierra y la segregación residencial". En: Revista EURE (online). Volumen XXVI, N ${ }^{0}$ 77. pp. 49-80. URL: http://www.scielo.cl/scielo.php?script=sci arttext\&pid=S0250-71612000007700003\&lng=es\&nrm=iso.

Sabatini, Francisco. 2003. La segregación social del espacio en las ciudades de América Latina. Documentos del Instituto de Estudios Urbanos y Territoriales, Serie Azul No 35. Pontificia Universidad Católica de Chile. Santiago de Chile. URL: http://www.territorioysuelo.org.

Velázquez, Guillermo y Rosana Cepeda. 2004. “Análisis de asociación espacial en variables de calidad de vida en la Argentina”. En: Revista Geográfica $N^{o} 136$. Instituto Panamericano de Geografía e Historia. Julio- diciembre de 2004. pp. 109-132.

Wong, David. 1993. "Spatial indices of segregation”. En: Urban Studies. Volumen 30. pp. 559572.

Wong, David. 1998. "Measuring multiethnic spatial segregation”. Urban Geography. Volumen 19. pp. 77-87. 